\title{
AQP-4 and its role in maintaining the hydric balance in the brain
}

\author{
Elena-Silvia Popescu', Daniel Pirici², Sabina Andrada Zurac ${ }^{3}$, Daniela Adriana Ion ${ }^{1}$ \\ ${ }^{1}$ Department of Physiopathology II, The Experimental Medicine and Fundamental Research \\ Laboratory, "Carol Davila" University of Medicine and Pharmacy, Bucharest, Romania \\ ${ }^{2}$ Department of Research Methodology, University of Medicine and Pharmacy of Craiova, Romania \\ ${ }^{3}$ Department of Pathology, "Carol Davila" University of Medicine and Pharmacy, \\ Colentina Clinical Hospital, Bucharest, Romania
}

\begin{abstract}
Aquaporins are a vast family of channel proteins whose main role is the bidirectional transport, depending on the osmotic gradient, of water across the lipid membranes, which have low permeability for this solvent. The aquaporins are also involved in the lipid metabolism, the cell proliferation and migration processes, the transport of glycerol, neuroexcitation and epithelial fluids secretion, having numerous roles such as ensuring the water transport in the central nervous system, the production of CSF, aqueous humor and saliva, epithelial hydration, urine concentration and nervous impulse transmission.

Up to now, in mammals there have been identified 13 types of aquaporins, each of them annotated from 0 to 12 (Aqp 0 - Aqp 12). Of these, aquaporin-4 (AQP-4) - located in the astroglia - is the most abundant aquaporin in the brain. Although this type of aquaporin is also present inside the brain parenchyma, especially in the astroglial processes lining the neuronal synapses, AQP-4 is mainly located in the astroglial end-feet adjacent to the ependymocyes or endothelial cells, where its main role is to ensure the bidirectional transport of water between the astroglia and the cerebrospinal fluid (CSF) or blood vessels. The presence of AQP-4 on the surface of astroglial processes promotes, in the initial stages of ischemia, the formation of the cytotoxic oedema, but has a protective role against vasogenic oedema. This review aims to describe the roles of AQP-4, and especially the role that this protein has in maintaining the hydric balance in the brain.
\end{abstract}

Keywords: AQP-4, hydric balance, astroglia

The aquaporins are a numerous channel protein family expressed in organisms from all kingdoms, up to the present time having been discovered, in plants, microorganisms, animals and humans, approximately 450 aquaporins $(1,2)$. Up to now, in mammals have been discovered 13 aquaporins (3), annotated, in the order of their discovery, from 0 to 12 , and classified, according to the transported substance, as aquaporins: AQP 0, AQP 1, AQP 2, AQP 4 and AQP 5 - transporting exclusively water, aquaglyceroporins: AQP 3, AQP 7, AQP 9 and AQP 10 - transporting water as well as glycerol, and "unorthodox" aquaporins: AQP 6, AQP 8, AQP 11 and AQP 12, the substance transported by these aquaporins not having been fully characterized so far (4). The aquaporins are small proteins, with a molecular weight of @ $30 \mathrm{kDa}(23481483)$ and 300 aminoacids in lenght (6), and have a common structure with 6 a-helical transmembrane domains (7) and 2 a-helical domains that don't fully pass through the cell membrane (5), domains which contain a consensus sequence composed of asparagine-proline-alanine (NPA) which enters the structure of a pore of @28 A $(1 \mathrm{~A}=0,1 \mathrm{~nm})(8,9)$, the amino- and carboxiterminal ends of these proteins being intracellular. (7)

Although most of the studies on this subject from the international literature attest that the first aquaporin (now known as AQP-1) was discovered in 1992 by Agre and his collaborators in the erythrocyte cell membrane (10), there is indisputable proof that its discovery took place much earlier, in 1988, by the Romanian scientist Gheorghe Benga (11). 
AQP-4 is a channel protein whose main role is the bidirectional transport, according to the osmotic gradient, of water across the lipid bistrate, which has low permeability for water and electrolytes. (10) AQP-4's selectivity for water is given by 3 specializations present along the monomeric AQP4 channel: the narrowing of the pore to @28 A - to prevent the entrance of molecules smaller than water, the presence of an arginine residue - which serves to block the entrance of protonated water and other cations, and the presence of positively charged dipoles in the middle portion on the channel - which are involved in preventing the flux of protons and in reorientating the water molecules (10).

Located mainly at the brain-blood and brainCSF barrier but also, to a lesser extent, inside the brain parenchyma, especially in the perisynaptic astroglial membrane portions, this channel-protein is one of the most abundant molecules in the brain. (10) It can also be localized - to a lesser extent, compared to its distribution in the brain - in other tissues, like the skeletal muscle, the renal collecting tubules, the gastric parietal cells, the respiratory epithelium and different glandular epithelia (12, 13,16).

Various studies using immunohistochemistry have shown that, in the CNS, AQP-4 is predominantly located in the brain astrocytes, with the biggest density in the astrocitary membranes which limit the cerebral microvessels or the pial layer (pia mater) on the surface of the brain, but its presence - albeit in smaller quantities - in the perisynaptic astrocyte processes reinforces the polarized distribution of this channel-protein in astrocytes (10).

Also in the CNS, but to a smaller extent compared to the astrocitary localization, the AQP-4 can also be found in the basolateral membranes of the ependymal cells, in the granular layer of the cerebellum and in the retina (15).

The extensions of the astrocyte membranes which contain the largest quantities of AQP-4 and are in contact with the endothelia of the cerebral capillaries are called end-feet (the density of AQP4 being, at this level, about @10 times higher compared to the rest of the astrocyte membrane (16) and are involved, besides ensuring the traffic of water through the lipid membranes of the astroglia, in different processes essential for maintaining the homeostasis in the CNS, such as nutrient exchange, water flow and possibly glucose and lactate metabolism (17).

The use of freeze-fracture electron microscopy techniques has revealed, on large areas of the astrocitary end-feet, the presence of proteic intramembranary aggregates. Discovered by Dermietzel (18), these aggregates have been named OAP (orthogonal arrays of particles), and their role has remained unknown until the observation that, in animals with knock-out for AQP-4, these aggregates were completely missing from the membranes of the end-feet. (19) Furthermore, after the transfection of ovarian cells with AQP-4 mRNA these proteic aggregates had appeared (20).

Due to the location of these proteic structures at the blood-brain and blood-CSF interface, the hypothesis of their role in maintaining the hydric balance in the brain has been launched, and it was presumed that they were involved in the control of the water flow between blood and the brain (10). This hypothesis has been confirmed by the observation that the extent of postischemic cerebral oedema was significantly decreased after the knock-out of AQP-4 (10,21,9,22).

These orthogonal proteic structures contain mainly the M23 isoform of AQP-4, which is considered one of the two classic isoforms of AQP-4. (23) Although, on the basis of the 6 types of AQP-4 c-DNA (produced by alternative splicing) discovered up to the present time at various species (and annotated from a to f), several AQP-4 isoforms have been described, the most frequent being AQP$4 \mathrm{M} 1$ and AQP-4 M23 (also found in humans), corresponding to the $\mathrm{AQP} 2 \mathrm{a}$, respectively $\mathrm{AQP} 4 \mathrm{c}$ cDNA(10).

The synthesis of these isoforms is encoded by the same gene, but the transcription initiation sites are different, leading to different structural and functional features. Thus, although both have the same water transport capacity, the isoform M23 (301 aminoacids), 22 aminoacids shorter than the M1 isoform (323 aminoacids) differs from the latter by its ability to polymerize, forming tetramers, grouped in orthogonal arrays of particles (OAPs). (10) Each of these tetramers has a central pore, which is thought to be involved in gas transport. (10) Thus, it was found (10) that, after oocyte transfection with AQP-4 mRNA, the membrane permeability for $\mathrm{CO}_{2}$ and $\mathrm{NH}_{3}$ had increased. 
Because the cell membrane permeability differs depending on the lipid composition, and the permeability level of the end-feet is still unknown, the degree of the involvment of OAPs in the gas transport has remained, up to the present time, unknown (10).

Observed through freeze fracture electron microscopy techniques, the OAPs are vast structures, with a density of @ 500-600/ $\mathrm{mm}^{2}$, that form a significant part of the blood brain barrier (1). Although the complexity and size of these structures doesn't justify their role in the transport of water in physiological processes, the role of OAPs could be explained by the necessity of a decrease in the level of cerebral oedema which appears in varied pathological processes produced in the CNS, like cerebral ischemia, trauma, various tumors, inflammatory processes or toxin exposure $(23,10)$.

Although, up to now, the mechanisms responsible for the production and clearance of cerebral oedema haven't been completely elucidated (10), in the oedema caused by ischemia (such as, for example, the oedema following an ischemic stroke) (23), the level of AQP-4 is, during the first days, constant, even diminished.(10) The presence of ischemia leads to the depletion of ATP and the cessation of functioning of the membrane $\mathrm{Na} / \mathrm{K}$ pump, the buildup of sodium ions inside the cell leading, subsequently, to the production of cytotoxic oedema through the influx of water in the cerebral parenchyma cells. This further leads to the swelling of the neurons and glia and the subsequent accumulation of an even larger quantity of water and electrolytes in the cerebral extracellular space $(23,24)$.

Thus, the presence of AQP-4 has an important role in the formation of cytotoxic oedema, as the water enters according to the osmotic gradient in the astroglia and neurons (25). Subsequently, the persistence of ischemia leads to the formation of vasogenic oedema, caused by the destruction of the endothelial tight junctions and the rupture of the blood-brain barrier, with the massive influx of water in the brain $(23,24)$, but, concurrently with this event, the up-reglation of AQP-4 contributes to the clearance of the cerebral oedema. Thus, in the vasogenic oedema, the presence of this channel protein has a protective role, decreasing the cerebral oedema and therefore decreasing the extension of neurological lesions (25). Furthermore, it was found that VEGF, a growth factor which is upreglated in ischemia, contributes to the increase of the AQP-4 level in this type of cerebral injury $(26,27)$.

Moreover, erythropoietin (EPO) has been found to have a neuroprotective role following ischemic oedema, possibly through this hormone's capacity to decrease the levels of AQP-4 (10).

Studies (22) on mice with knock-out for AQP-4 have proven the existence, at these animals, of a reduced level of cytotoxic oedema and a high recovery rate of the neurological lesions produced by water poisoning, focal cerebral ischemia and bacterial meningitis. In addition, in mice lacking AQP-4 which had induced vasogenic oedema (which can be produced though cortical freeze, brain tumour, brain abscess and hydrocephalus), the level of oedema was more increased and the clinical outcomes, worse (22).

The modulation of AQP-4, with its down-regulation during the first phase of ischemia and its upregulation during the late phase of this process could, thus, represent a therapeutic target in ishemic stroke $(25,28)$.

AQP-4 is tethered to the astrocyte membrane through the DAPC complex (dystrophin-associated protein complex)(10)(Fig. 1), which has the role of anchoring the cytoskeleton to the extracellular matrix (30). Thus, the absence of different component members of DAPC, such as dystrophin, a-syntrophin, b-dystroglycan, a-dystrobrevin or laminin (30), leads to the loss of AQP-4 polarity and the destruction of OAPs. This explains the low level of AQP-4 in the Duchenne and Becker dystrophy, two dystrophinopathies with an X-linked inheritance pattern caused by mutations in the DMD gene, which encodes dystrophin (31).

Dystrophin is one of the main components of DAPC (dystrophin-associated protein complex), a complex located beneath the basal lamina which extends into the intracellular space, within the cytoplasm. Dystrophin has the role of connecting the cytoskeleton (by binding $\mathrm{F}$-actin to its $\mathrm{N}$-terminal domain) to the extracellular matrix (by binding a-dystrobrevins, syntrophins and b-dystroglycan to its C-terminal domain). b-dystroglycan binds through its extracellular domain -to a-dystroglycan, which in turn binds to laminin, a component of the basal membrane, which binds further to collagen. 


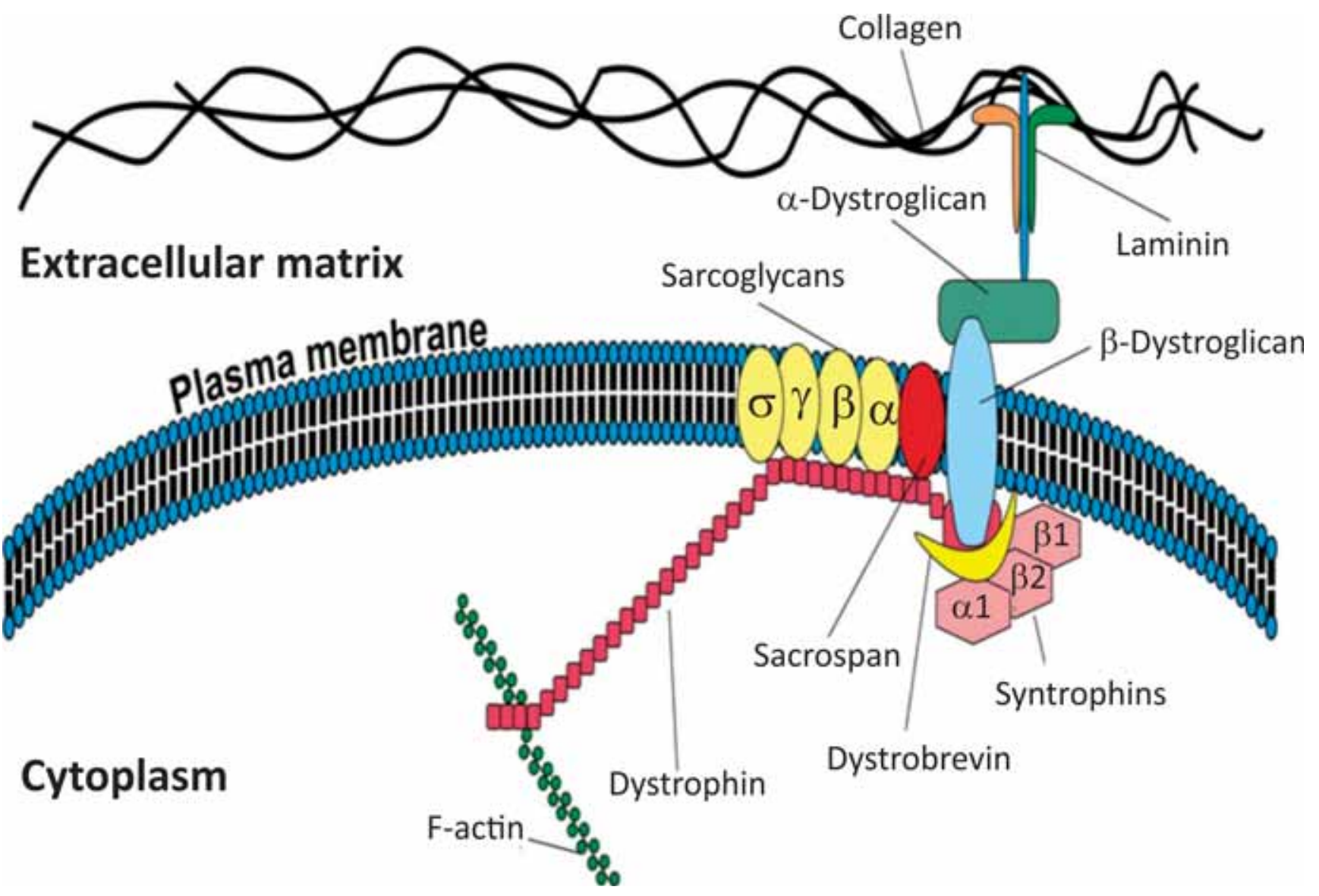

FIGURE 1. The dystrophin-associated protein complex.

Furthermore, dystrophin indirectly binds, through dystrobrevin, to the sarcoglycan-sarcospan complex.

Nevertheless, it has been found that the knockout of a-syntrophin or dystrophin doesn't lead to the complete lack of AQP-4 expression, as this protein continues to be expressed, albeit in lower quantities, after the deletion of the genes for these members of the DAPC complex (10).

However, the absence of AQP-4 doesn't lead to the disintegration of the DAPC complex: in one of the studies on AQP-4 it was found that in the astrocytes with knock-out for AQP-4, the actin fibers were depolymerized, but the levels of dystrophin, a-syntrophin and b-dystroglycan (all of which are components of DAPC) remained unchanged (17).

It was also found that AQP-4 is colocalised with the K channels Kir4.1, both of these channels being associated to DAPC (dystrophin-associated protein complex) (17,32).

Recently, a transcript of AQP-4 which doesn't contain the exon 4 has been identified, the protein encoded by it having been annotated AQP-4-D4. The transfection of AQP-4-D4 in astrocytes expressing functional AQP-4 was found to lead to a reduction of the surface expression of this channel protein and to the decrease of water transport across the cell membrane, compared to the $\mathrm{AQP}-4^{+++}$astrocytes which weren't transfected with AQP-4-D4. It was thus considered that the down-regulation of the expression and activity as a water channel of AQP4 was caused by the formation of heterodimers between AQP-4 and AQP-4-D4 (33).

AQP-4 is also involved in the neuroinflammation process, which appears in many cerebral injuries, both acute and chronic (like, for example, multiple sclerosis) and is associated with the destruction of the blood brain barrier and the formation of oedema (9).

The main cellular alteration process which appears in neuroinflammation is the activation of microglia and astrocytes. Following activation, the microglia - branched cells when they are not activated (24) - lose their extensions, becoming round, 
and start to produce both proinflammatory cytokines, such as ca TNF- $\alpha$, IL- $1 \beta$ and IL- 6 and anti inflammatory cytokines, such as TGF- $\beta$, IL-4 and IL-10 (9).

Moreover, the astrocytes are activated through water influx leading to their subsequent hypertrophy. This process facilitates their movement using rolling, with the help of cytoplasmic extensions called lamellipodia, towards the brain lesion, where they contribute to the tissue repair through astrogliosis (9).

In vitro, the absence of $\mathrm{AQP}-4$ delays the astrocyte migration appeared in response to a chemotactic stimulus, and in vivo, the deletion of AQP-4 hinders the formation of the glial scar which is in fact the main response of the brain parenchyma to almost any lesion (22).

Although the astrogliosis process can be beneficial, the astrocytes' capacity of movement can also have disadvantages: the tumoral cells of the astrocytoma express high quantities of AQP-4, which can facilitate their infiltration in the brain(5).

Recent data (9) suggests the existence of a relationship between AQP-4 and microglial activation. Thus, studies on mice with knock-out for AQP-4 have shown that in these animals the activation of the astrocytes and the astrogliosis process have been deemed inversely proportional to the microglial activation. The mechanisms explaining the decrease of the astrocitar level proportional to the microglial activation could be explained by the presence, on the microglial cell membrane surface, of stretch chloride channels, which are activated by osmotic stress. It was found that the activation of these channels contributes to the maintenance of microglia in an inactive state (being, thus, branched). The inhibition or deletion of AQP-4 will thus lead to the increase of extracellular fluid and the decrease of osmotic pressure at this level, with the inactivation of the stretch chloride channels and the subsequent activation of the microglia (9).

The activated microglia secrete matrix-metalloproteinases, proteolytic enzymes involved in the remodeling of the extracellular matrix (29), which lead to the increase in the level of vasogenic oedema through the degradation of the basal lamina between the end-feet and the endothelium and the destruction of the OAPs with the loss of astrocyte polarity, this also contributing to the maintenance of the inverse proportionality relationship between microglia and astrocytosis. Thus, MMP9 and MMP2 degrade agrin, and MMP-3 degrades dystroglycan, two proteins which are crucial in maintaining the OAPs' structure (9).

A connection between AQP-4 and neuroinflammation could be optic neuromyelitis, a demyelinating disease characterized by the presence of anti-AQP-4 and anti-OAP G immunoglobulines (9). Moreover, it was found that the myasthenia gravis patients also present anti-AQP-4 antibodies, but so far the relationship between myasthenia gravis and neuroinflammation has not been fully understood. Still, the hypothesis of a common autoimmune origins of these diseases has not been excluded (9).

Besides protecting against vasogenic oedema through its upregulation in ischemia, other roles AQP-4 plays in the maintenance of the hydric balance in the brain are the reduction of the neuroinflammation process (and hence the reduction in the production of vasogenic oedema through the maintainance of inactive microglia), the buffering of potassium (through the potassium receptor Kir4.1 which functions in close connection to AQP-4), and the role of regulating the perisynaptic volume, which increases in level following the nervous impulse (10).

Thus, the extracellular water, which has been accumulated after the electrical impulse discharge, is directed towards the astroglia, and enters these cells through AQP-4 (10).

Regarding the role of AQP-4 in maintaining the hydric balance in the brain, a theory has been formulated, according to which AQP-4 is involved in the clearance of toxic metabolites, its absence being correlated with the apparition of neurodegenerative diseases, such as Alzheimer or Parkinson. Up to now, this theory has not yet been confirmed (10).

AQP-4 has also been involved in the production of epileptic seizures. Thus, it was found that in knock-out animals with knock-out for this protein the severity of the seizures was more important, but the threshold was higher, compared to the animals which were not expressing AQP-4. This can be explained by the increase, at mice which do not express AQP-4, of the extracellular volume and the dilution, as a result, of the potassium existing in the perisynaptic excesses water, with the reduction of potassium reuptake (10). 
AQP-4 is also expressed in the sensory organs. Thus, it is present in the Muller retinal cells (its absence leading to subtle alterations in the electroretinogram) or in the internal ear (its lack leading to impaired hearing, which can be explained by the osmotic caliber alterations in the Corti organ, caused by its absence) (10).

Moreover, AQP-4 is also involved in the regulation of the nervous impulse transmission, such as the regulation of the excitability. Thus, the absence of AQP-4 decreases, in the brain, the level of theta waves, which are dependent on the brain-derived neurotrophic factor (BDNF) and the receptor for its tyrosin-kinase (TrkB). The cause for which the theta wave potential is dependent on AQP-4 hasn't been understood yet, but it is thought that the lack of AQP-4 decreases the level of neurotrophins. Thus, in mice which do not express AQP-4 there have been recorded long periods of depression, which has been neutralized after the removal of the brain-derived neurotrophic factor (BDNF) (10).

\section{REFERENCES}

1. Cui J., Bastien D.A. Molecular Dynamics Simulation and Bioinformatics Study on Yeast Aquaporin Aqy1 from Pichia Pastoris. Int J Biol Sci. 2012; 8(7): 1026-1035

2. Benga G. Water Channel Proteins (Later Called Aquaporins) and Relatives: Past, Present and Future. Life. 2009; 61(2): 112-133

3. Ching-Hei Jeung. Aquaporins in spermatozoa and testicular germ cells: identification and potential role. Asian J Androl. 2010; 12: 490-499

4. Palmgren M. Regulation and Transport Mechanisms of Eukariotic Aquaporins. Doctoral Thesis. Department of Chemistry and Molecular Biology, Microbiology, University of Gothenburg, Sweden. 2013

5. Papadopoulos M.C., Verkman A.S. Aquaporin water channels in the nervous system. Nat Rev Neurosci. 2013; 14(4):265-277.

6. Sorani M. The Role of Genetic Variation in Aquaporin-4 on Therapeutic and Physiological Response After Traumatic Brain Injury. 2007. ProQuest. ISBN: 0549333150,9780549333159

7. Gohar O. Aquaporins: The Waterways of Nature. Modulator. 2010; 24: 4-9.

8. Verkman A.S. Aquaporins at a glance. J Cell Sci. 2011; 124: 2107-2112

9. Fukuda A.M., Badaut J. Aquaporin 4: A Player in Cerebral Edema and Neuroinflammation. J Neuroinflammation. 2012; 9 (279)

10. Nagelhus E.A., Ottersen O.P. Physiological Roles of Aquaporin-4 in Brain. Physiol Rev. 2013; 93(4):1543-1562

11. Benga G. Water transport in red blood cell membranes. Prog. Byophis. Molec. Biol., 51, 193-245, 1988.

12. Verkman A.S., Ratelade J., Rossi A., Zhang H. et al. Aquaporin-4: Orthogonal Array Assembly, CNS Functions, and Role in Neuromyelitis Optica. Acta Pharmacol Sin. 2011; 32: 702-710

13. Yool A.J., Brown E.A., Flynn G.A. Roles for novel pharmacological blockers of aquaporins in the treatment of brain oedema and cancer. Clin Exp Pharmacol Physiol. 2010; 37(4): 403-9.
Furthermore, although the mechanisms responsible for these processes have not been elucidated yet, it was found that the lack of this water channel led to impaired locative memory (10).

Although the most abundant, AQP-4 is not the only channel protein in the brain, at this level being present, albeit in a smaller quantity, other aquaporins such as AQP-1, AQP-9 or AQP-11, factors which must be taken into account in the analysis and modulation of the process of maintaining the hydric balance in the brain (10).

\section{Acknowledgements}

The authors would like to thank their colleagues from the The Experimental Medicine and Fundamental Research Laboratory, Department of Physiopathology II, „Carol Davila” University of Medicine and Pharmacy, Bucharest, Romania and from the Department of Research Methodology and Immunohistochemistry, University of Medicine and Pharmacy of Craiova, Romania, for their support.

Conflict of interest: none declared Financial support: none declared

14. Benarroch E.E. Aquaporin-4, homeostasis, and neurologic disease. Neurology. 2007; 69(24): 2266-8

15. Nicchia G.P., Rossi A., Mola M.G., Pisani F. et al. Higher order structure of aquaporin-4. Neuroscience. 2010; 168(4): 903 - 14.

16. Nagelhus E.A., Veruki M.L., Torp R., Haug F.M. et al. Aquaporin-4 Water Channel Protein in the Rat Retina and Optic Nerve: Polarized Expression in Muller Cells and Fibrous Astrocytes. J Neurosci. 1998; 18(7): 2506-19

17. Gundersen G.A. (2013). Roles of aquaporin-4 in brain fluid dynamics. PhD. Thesis. University of Oslo: Norway.

18. Noell S., Wolburg-Buchholz K., Mack A.F., Beedle A.M. et al. Evidence for a Role of Dystroglycan Regulating the Membrane Architecture of Astroglial Endfeet. EJN. 2011; 33: 2179-2186

19. Verbavatz J.M., Ma T., Gobin R., Verkman A.S. Absence of Orthogonal Arrays in Kidney, Brain and Muscle from Transgenic Knockout Mice Lacking Water Channel Aquaporin-4. J Cell Sci. 1997; 110: $2855-2860$

20. Jang B., Brown D., Verkman A.S. The Mercurial Insensitive Water Channel (AQP-4) Forms Orthogonal Arrays in Stably Transfected Chinese Hamster Ovary Cells. J Biol Chem. 1996; 271(9): 4577-80

21. Manley G.T., Fujimura M., Ma T., Noshita T., et al. Aquaporin-4 Deletion in Mice Reduces Brain Edema after Acute Water Intoxication and Ischemic Stroke. Nat Med. 2000; 6(2): 159-63

22. Verkman A.S., Binder D.K., Bloch O., Auguste K., et al. Three distinct roles of aquaporin-4 in brain function revealed by knockout mice. Biochim Biophys Acta. 2006; 1758: 1085-1903.

23. Liang D., Bhatta S., Gerzanich, Simard J.M. Cytotoxic Edema. Mechanisms of Pathological Cell Swelling. Neurosurg Focus. 2007; 22(5): E2.

24. Mogoanta L., Ciurea M. Pirici I., Margaritescu C., Simionescu C., Ion D.A., Pirici D. Different Dynamics of Aquaporin 4 and Glutamate Transporter-1 Distribution in the Perineuronal and Perivascular Compartmentsduring Ischemic Stroke. Brain Pathol. 2014; 24(5): 475-93 
25. Tourdias T., Mori N., Dragonu I., Cassagno N. et al. Differential aquaporin 4 expression during edema build-up and resolution phases of brain inflammation. JNeuroinflammation. 2011; 8:143.

26. Rite I., Mackado A., Cano J., Venero J.L. Intracerebral VEGF Injection Highly Upregulates AQP4 mRNA and Protein in the Perivascular Space and Glia Limitans Externa. Neurochem Int. 2008; 52(4-5): 897-903

27. Chu H., Tang Y., Dong Q. Protection of Vascular Endothelial Growth Factor to Brain Edema Following Intracerebral Hemorrhage and Its Involved Mechanisms: Effect of Aquaporin-4. Plos One. 2013.

28. Zador Z., Stiver S., Wang V., Manley G.T. Role of aquaporin-4 in cerebral edema and stroke. Handb.Exp.Pharmacol. 2009; (190): 159-70.

29. Maeda A., Sobel R.A. Matrix Metalloproteinases in the Normal Human Central Nervous System, Microglial Nodules, and Multiple Sclerosis Lesions. J Neuropathol Exp Neurol. 1996; 55(3): 300-9
30. Ehmsen J., Poon E., Davies K. The dystrophin-associated protein complex. J Cell Sci. 2002; 115:2801-2803

31. Darras B.T., Miller D.T., Urion D.K. Dystrophinopathies. Gene Reviews. 2000.

32. Connors N.C., Adams M.E., Froehen S.C., Kofuji P. The Potassium Channel Kir4.1 Associates with the Dyystrophin-Glycoprotein Complex via a-Syntrophin in Glia. JBC. 2004; 279: 28387-28392.

33. De Bellis M., Pisani M., Mola M.G., Basco D. et al. A novel human aquaporin-4 splice variant exhibits a dominant-negative activity: a new mechanism to regulate water permeability. MBOC. 2014; 25(4): 470-480. 